Electronic Supplementary Material (ESM)

2 Concentrations and long term temporal trends of hexabromocyclododecanes (HBCDD) in lake trout and walleye from the Great Lakes

4 Bita Alipour Parvizian, ${ }^{\text {a }}$ Chuanlong Zhou, ${ }^{\mathrm{b}}$ Sujan Fernando, ${ }^{\mathrm{c}}$ Bernard S. Crimmins, ${ }^{\mathrm{d}}$ Philip K. Hopke, ${ }^{\mathrm{c}}$,

5 e Thomas M. Holsen ${ }^{\mathrm{a}, \mathrm{c}}$

$6 \quad{ }^{a}$ Civil and Environmental Engineering, Clarkson University, Potsdam, NY

$7 \quad$ bchool of Environment, Guangzhou Key Laboratory of Environmental Exposure and Health, and

8 Guangdong Key Laboratory of Environmental Pollution and Health, Jinan University, Guangzhou, China

$9 \quad{ }^{\mathrm{c}}$ Center for Air Resources Engineering and Science, Clarkson University, Potsdam, NY

10 dAEACS, LLC, New Kensington, PA 
26 Extraction method S2

27 Age normalization S3

28 Measured concentrations S6

29 HBCDD diastereomer distribution similarities within the Great Lakes S12

\section{$30 \quad$ Tables}

31 Table S1. Most correlated PBT to $\sum$ HBCDD for each lake. S5

32 Table S2. Age normalization factors for each lake and year. S5

33 Table S3. Tukey-Kramer analysis for $\sum$ HBCDD concentration comparison within the lakes. S6

34 Table S4. Lake Michigan measured concentrations for each HBCDD diastereomer and $\sum H B C D D . \quad$ S7

35 Table S5. Lake Ontario measured concentrations for each HBCDD diastereomer and $\sum$ HBCDD. $\quad$ S8

36 Table S6. Lake Superior measured concentrations for each HBCDD diastereomer and $\sum$ HBCDD. $\quad$ S9

37 Table S7. Lake Huron measured concentrations for each HBCDD diastereomer and $\sum$ HBCDD.

38 Table S8. Lake Erie measured concentrations for each HBCDD diastereomer and $\sum$ HBCDD. $\quad$ S11

39 Table S9. HBCDD diastereomer distribution similarities between the Great Lakes. S12

40 Table S10. Tukey-Kramer analysis for $\alpha$-HBCDD distribution similarities between the Great Lakes. S12

41 Table S11. Tukey-Kramer analysis for $\beta$-HBCDD distribution similarities between the Great Lakes. S12

42 Table S12. Tukey-Kramer analysis for $\gamma$-HBCDD distribution similarities between the Great Lakes. S13

43 Figures

44 Figure S1. Relative recovery of ${ }^{13} \mathrm{C}$ spiked $\alpha$-HBCDD using ASE under different conditions. S3

45 Figure S2. Stability test of each diastereomer under ASE extraction conditions. S3

46 Figure S3. Fish age trends. S4

47 Figure S4. A schematic of age normalization procedure for $\sum$ HBCDD. S5

\section{Extraction method}

50 Several tests using two different mobile phase, acetone:hexane $(1: 1 \mathrm{v} / \mathrm{v})$ and methylene chloride, in 51 three different temperatures, 60,90 , and $125^{\circ} \mathrm{C}$, were carried out to optimize the extraction method.

52 Prepared samples for these tests contained $0.75 \mathrm{~g}$ of drying agent sodium polyacrylate, spiked with $10 \mathrm{ng}$ 53 of ${ }^{13} \mathrm{C}$ labeled $\alpha$-HBCDD. Relative recovery of ${ }^{13} \mathrm{C}$ labeled $\alpha$-HBCDD was calculated (surrogate peak 54 area/ reference peak area) and shown in Figure S1. Methylene chloride showed higher recovery results 55 compared to acetone/hexane, and lower temperature compared to $125^{\circ} \mathrm{C}$, which is the temperature used in 56 previous studies by GLFMSP for chemicals such as PBDEs and PCBs. ${ }^{1}$ 


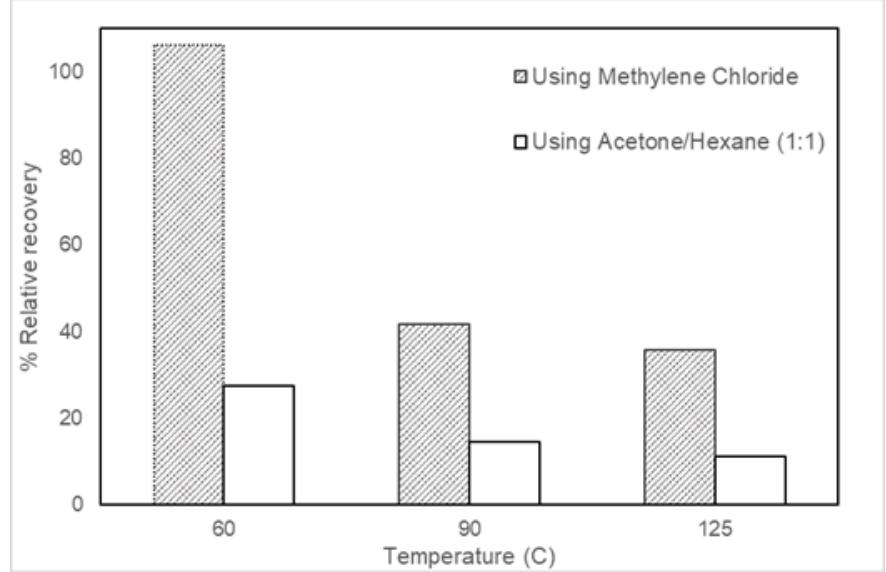

58 Figure S1. Relative recovery (peak area in sample/peak area in reference) of ${ }^{13} \mathrm{C}$ labeled $\alpha$-HBCDD in

59 samples extracted using methylene chloride and acetone/hexane in different temperatures.

60 The stability of the HBCDD diastereomers under finalized ASE condition used for sample extraction was 61 tested using separate ASE cells spiked with $10 \mathrm{ng}$ of each HBCDD diastereomer. After extraction each 62 sample contained only one diastereomer and no trace of the others (Figure S2) proving that these 63 diastereomers are stable under the experiment procedure used.
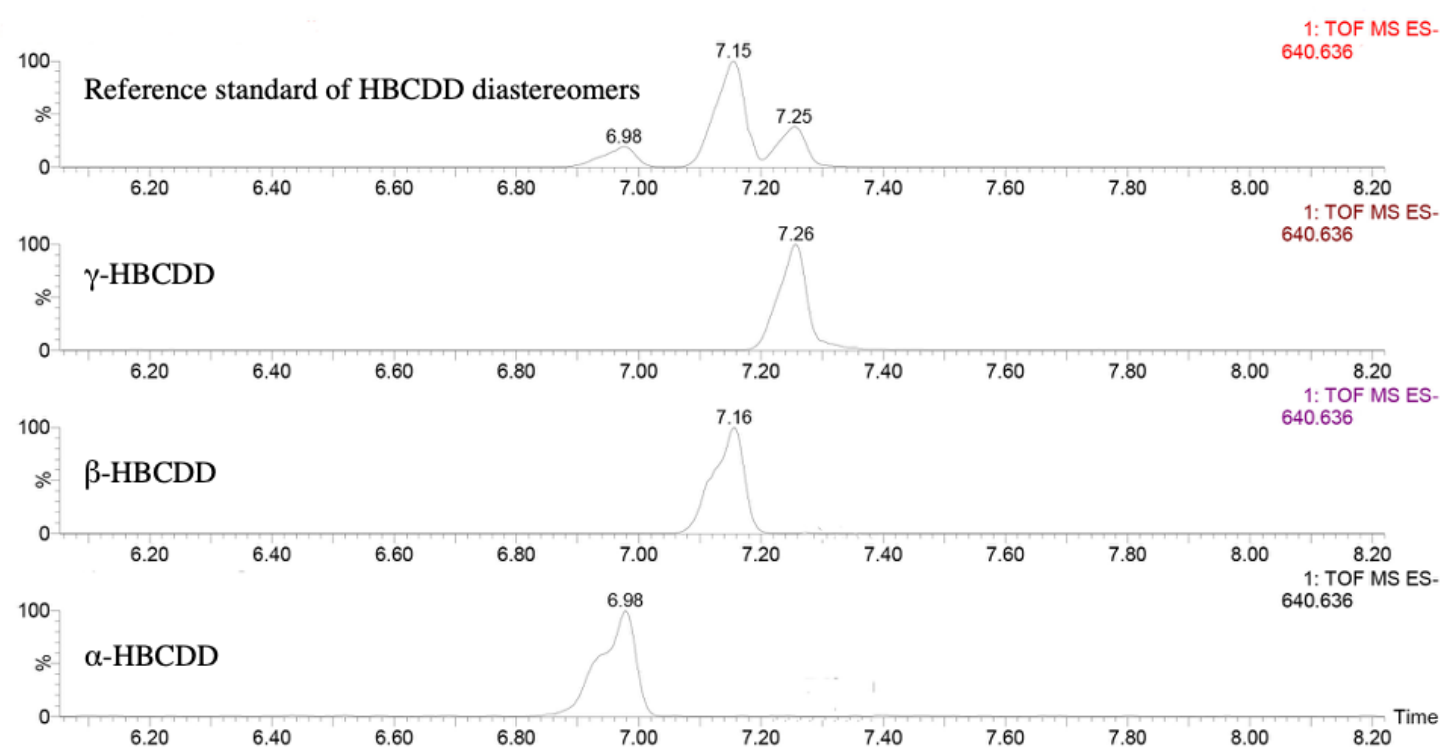

64

65 Figure S2. Stability test of each diastereomer under ASE extraction conditions. 

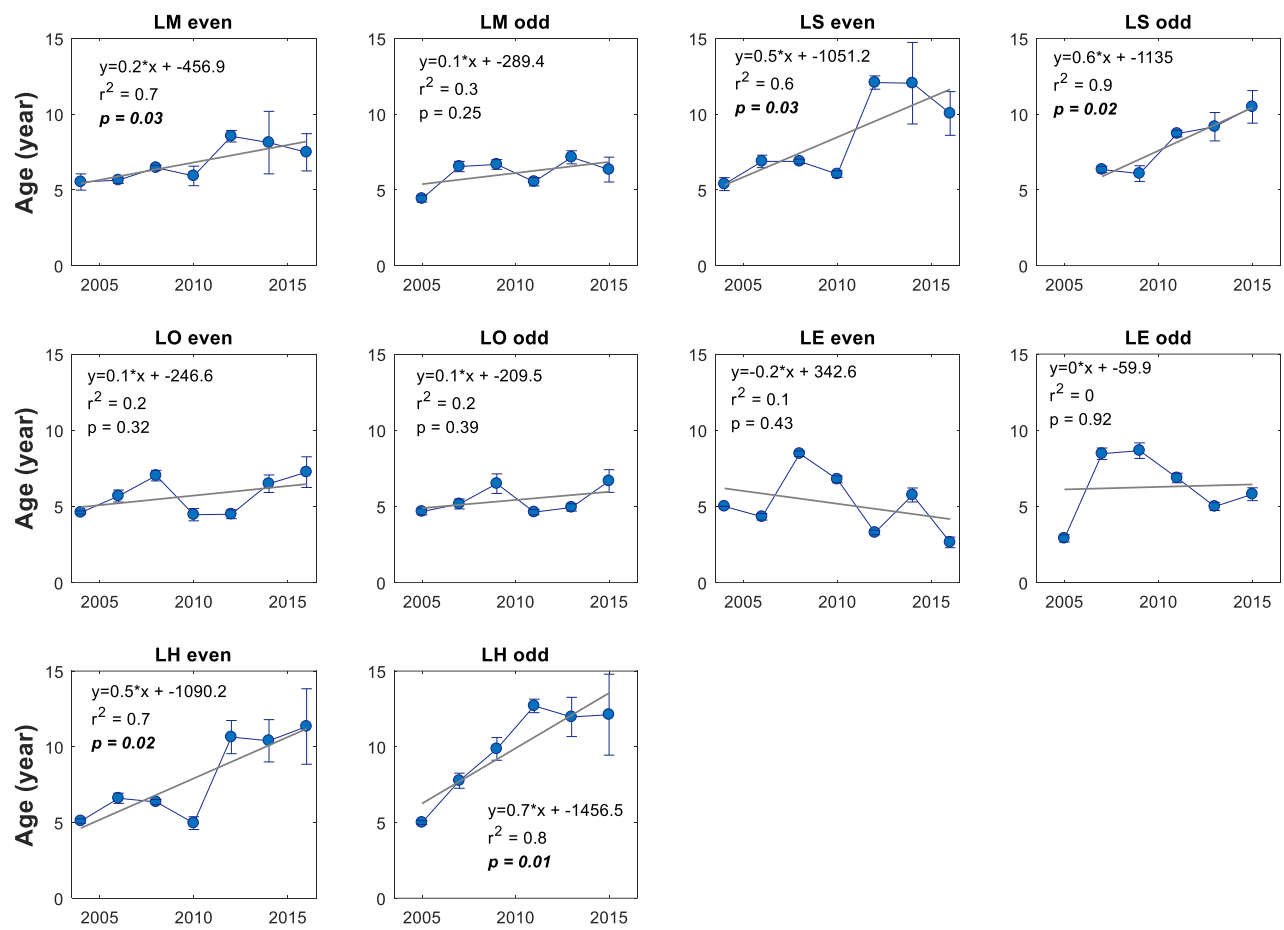

Figure S3. Age trends of specified size fish composites collected at each site from 2004 to 2016 (the $p$ values in bold and italic format indicate the increasing trends are significant at $p<0.05$ ). The age of a fish composite represents an average age of five individual fish homogenized into that composite. Error bars represent the standard deviation of the calculated ages of 10 composites.

72 Age normalization for this study follows these steps. Using 50 fish samples collected (same size, known age) 10 homogenized composite sample containing 5 fish and one homogenized mega sample containing 50 fish are made. In previous studies by GLFMSP concentration of several PBT in the 10 composites are quantified. $\sum$ HBCDD concentration using mega samples for each lake and year was quantified and most correlated PBT with data set is determined using hierarchical clustering for different years. Using the steps mentioned in Zhou et al. $2017^{2}$ age normalized concentration for the selected PBT is determined and applied to $\sum$ HBCDD results. Normalization factors are calculated using the ratio of original concentration and normalized concentration. A schematic of age normalization procedure for mega composite samples is presented in Figure S4. 


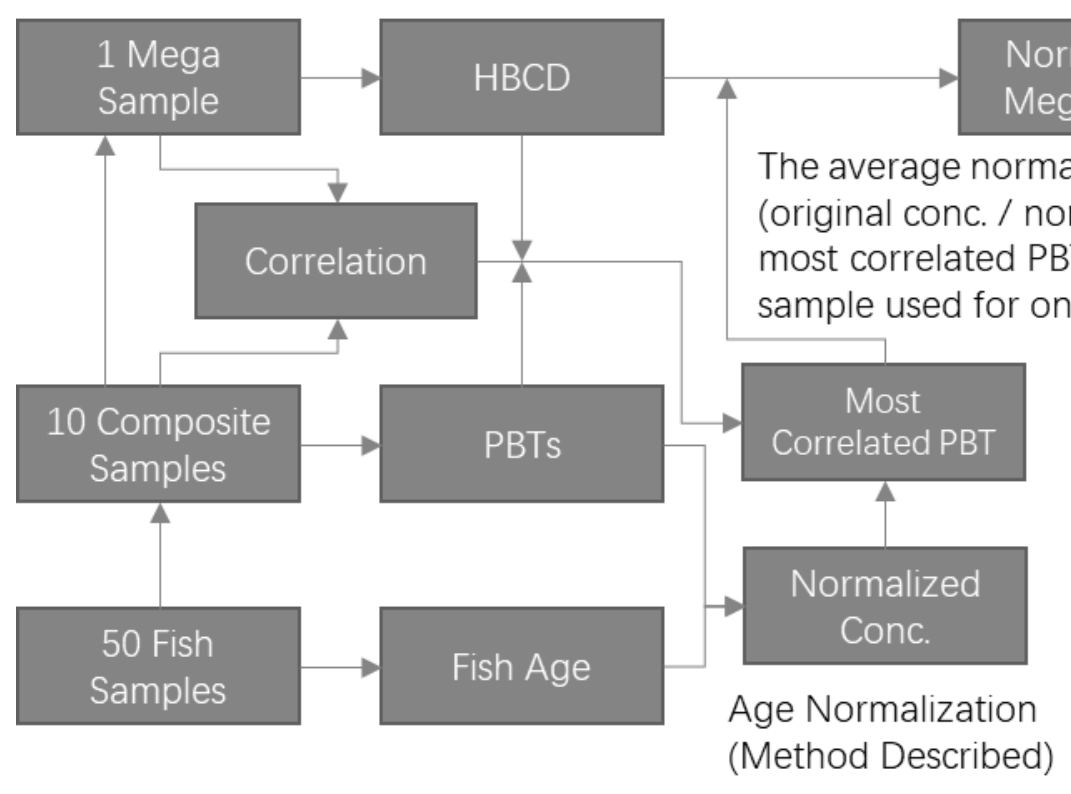

(Method Described)

82 Figure S4. Schematic description of the age normalization of $\sum H B C D D$ concentrations in fish mega-

83 composites. $\mathrm{PBT}=$ persistent, bioaccumulative, and toxic chemical.

84 Because of not having a constant ratio of diastereomers from year to year, age normalization for

85 HBCDDs was carried out based on the $\sum$ HBCDDs. Table S1 shows the most correlated PBT and

86 correlation coefficient and $\mathrm{p}$ value (bold means significantly correlated) for $\sum$ HBCDDs for each lake.

87 Table S2 presents normalization factors for each lake and year.

88 Table S1. Most correlated PBT to $\sum$ HBCDD with correlation coefficient and p value result.

\begin{tabular}{|l|l|l|}
\hline Lake & PBT & Correlation coefficient, p value \\
\hline Michigan & Endrin & $0.63,0.04$ \\
\hline Ontario & Tot-DDTs & $0.70,0.02$ \\
\hline Superior (even) & BDE_100 & $0.97,0.00$ \\
\hline Superior (odd) & Dieldrin & $0.61,0.39$ \\
\hline Huron & BDE_154 & $0.54,0.05$ \\
\hline LE & HCB & $0.34,0.31$ \\
\hline
\end{tabular}

89 
Table S2. Age normalization factors for each lake and year using $\sum$ HBCDD measured concentrations.

\begin{tabular}{|c|c|c|c|c|c|}
\hline Year & Lake Michigan & Lake Ontario & Lake Superior & Lake Huron & Lake Erie \\
\hline 2004 & 0.66 & 1.27 & 0.93 & 0.96 & 0.91 \\
\hline 2005 & 0.73 & 0.66 & 1.00 & 0.85 & 0.51 \\
\hline 2006 & 0.87 & 0.81 & 1.13 & 1.04 & 0.88 \\
\hline 2007 & 0.95 & 0.71 & 0.95 & 1.30 & 1.43 \\
\hline 2008 & 0.96 & 1.04 & 1.13 & 1.07 & 1.08 \\
\hline 2009 & 0.98 & 0.95 & 0.91 & 1.65 & 1.48 \\
\hline 2010 & 0.90 & 0.62 & 1.02 & 0.88 & 1.00 \\
\hline 2011 & 0.71 & 0.61 & 1.65 & 2.12 & 1.00 \\
\hline 2012 & 1.77 & 0.82 & 4.05 & 1.59 & 0.68 \\
\hline 2013 & 1.12 & 0.84 & 1.80 & 2.00 & 1.04 \\
\hline 2014 & 1.62 & 0.98 & 4.02 & 1.56 & 0.85 \\
\hline 2015 & 1.12 & 0.84 & 1.80 & 2.00 & 1.04 \\
\hline 2016 & 1.62 & 0.98 & 1.09 & 1.56 & 0.85 \\
\hline
\end{tabular}

93 Measured concentration for each lake and year ( \pm standard deviation) are presented in tables S4, S5, S6, 94 S7, and S8.

95 Table S3. $\sum$ HBCDD concentration (2004-2016) Tukey-Kramer analysis within lakes after Kruskal96 Wallis One-way ANOVA on ranks tests with $\mathrm{p}<0.001$.

\begin{tabular}{|l|l|}
\hline Lakes & Tukey-Kramer, $p$ value \\
\hline
\end{tabular}




\begin{tabular}{|l|l|}
\hline LM vs LO & 0.88 \\
\hline LM vs LH & 0.31 \\
\hline LM vs LS & 0.15 \\
\hline LM vs LE & $<0.05$ \\
\hline LO vs LH & $<0.05$ \\
\hline LO vs LS & $<0.05$ \\
\hline LO vs LE & 0.06 \\
\hline LH vs LS & 0.99 \\
\hline LH vs LE & $<0.001$ \\
\hline LS vs LE & $<0.001$ \\
\hline
\end{tabular}

97

98 Table S4. Lake Michigan measured concentration of each diastereomer and $\sum$ HBCDDs in ng/g ww.

\begin{tabular}{|l|l|l|l|l|l|}
\hline $\begin{array}{l}\text { Lake } \\
\text { Michigan }\end{array}$ & Year & $\alpha-$ HBCDD & $\beta$-HBCDD & $\gamma$-HBCDD & $\sum$ HBCDDs \\
$(\mathrm{ng} / \mathrm{g} w w)$ & $(\mathrm{ng} / \mathrm{g} w w)$ & $(\mathrm{ng} / \mathrm{g}$ ww $)$ & \\
\hline 1978 & $1.91 \pm 0.26$ & $1.48 \pm 0.48$ & $0.52 \pm 0.59$ & $3.91 \pm 0.43$ \\
\hline 1986 & $2.55 \pm 0.56$ & $1.54 \pm 0.49$ & $0.92 \pm 0.71$ & $5 \pm 0.79$ \\
\hline 1992 & $4.28 \pm 0.35$ & $0.84 \pm 0.10$ & $2.26 \pm 0.10$ & $7.39 \pm 0.82$ \\
\hline 2004 & $6.03 \pm 0.09$ & $0.80 \pm 0.00$ & $2.12 \pm 0.29$ & $8.95 \pm 0.27$ \\
\hline 2005 & $6.93 \pm 1.40$ & $0.78 \pm 0.18$ & $1.30 \pm 1.05$ & $9.01 \pm 1.39$ \\
\hline 2006 & $3.22 \pm 0.60$ & $1.28 \pm 1.03$ & $0.21 \pm 0.22$ & $4.71 \pm 0.23$ \\
\hline 2007 & $3.57 \pm 0.82$ & $1.29 \pm 1.04$ & $0.15 \pm 0.25$ & $5.01 \pm 0.20$ \\
\hline 2008 & $3.80 \pm 0.14$ & $1.28 \pm 0.84$ & $1.17 \pm 0.72$ & $6.24 \pm 1.41$ \\
\hline
\end{tabular}




\begin{tabular}{|l|l|l|l|l|}
\hline 2009 & $4.66 \pm 0.50$ & $0.59 \pm 0.11$ & $0.61 \pm 0.61$ & $5.86 \pm 1.00$ \\
\hline 2010 & $4.29 \pm 1.10$ & $1.28 \pm 1.03$ & $0.21 \pm 0.37$ & $5.78 \pm 0.61$ \\
\hline 2011 & $3.66 \pm 1.43$ & $1.26 \pm 1.02$ & $0.18 \pm 0.31$ & $5.11 \pm 0.88$ \\
\hline 2012 & $5.42 \pm 0.83$ & $0.60 \pm 0.30$ & $0.76 \pm 0.28$ & $6.78 \pm 1.09$ \\
\hline 2013 & $4.78 \pm 0.16$ & $0.62 \pm 0.30$ & $0.87 \pm 0.50$ & $6.27 \pm 0.96$ \\
\hline 2014 & $5.73 \pm 0.43$ & $1.78 \pm 0.01$ & $0.57 \pm 0.01$ & $8.11 \pm 0.88$ \\
\hline 2015 & $4.65 \pm 0.10$ & $0.74 \pm 0.05$ & $1.29 \pm 1.59$ & $6.68 \pm 1.74$ \\
\hline 2016 & $5.89 \pm 0.06$ & $0.76 \pm 0.01$ & $0.51 \pm 0.03$ & $7.16 \pm 0.11$ \\
\hline
\end{tabular}

99

100 Table S5. Lake Ontario measured concentration of each diastereomer and $\sum$ HBCDDs in ng/g wet weight.

\begin{tabular}{|c|c|c|c|c|c|}
\hline $\begin{array}{l}\text { Lake } \\
\text { Ontario }\end{array}$ & Year & $\begin{array}{l}\alpha-H B C D D \\
(n g / g ~ w w)\end{array}$ & $\begin{array}{l}\beta-\mathrm{HBCDD} \\
(\mathrm{ng} / \mathrm{g} \mathrm{ww})\end{array}$ & $\begin{array}{l}\gamma \text {-HBCDD } \\
(n g / g w w)\end{array}$ & $\begin{array}{l}\sum \text { HBCDDs } \\
\text { (ng/g ww) }\end{array}$ \\
\hline 1978 & & $3.50 \pm 0.43$ & $1.65 \pm 0.12$ & $2.47 \pm 0.42$ & $7.31 \pm 0.56$ \\
\hline 1986 & & $3.08 \pm 0.21$ & $1.37 \pm 0.04$ & $1.50 \pm 0.12$ & $5.95 \pm 0.30$ \\
\hline 1992 & & $3.03 \pm 0.21$ & $1.35 \pm 0.01$ & $1.35 \pm 0.01$ & $5.74 \pm 0.19$ \\
\hline 2004 & & $3.36 \pm 0.22$ & $0.36 \pm 0.15$ & $1.39 \pm 0.44$ & $5.11 \pm 0.58$ \\
\hline 2005 & & $4.29 \pm 0.23$ & $0.86 \pm 0.76$ & $3.75 \pm 2.96$ & $8.90 \pm 3.62$ \\
\hline 2006 & & $4.73 \pm 0.37$ & $1.19 \pm 0.70$ & $2.11 \pm 0.91$ & $8.04 \pm 1.93$ \\
\hline 2007 & & $3.64 \pm 0.45$ & $0.33 \pm 0.03$ & $0.74 \pm 0.15$ & $4.71 \pm 0.58$ \\
\hline 2008 & & $4.69 \pm 0.43$ & $0.20 \pm 0.11$ & $0.43 \pm 0.22$ & $5.31 \pm 0.75$ \\
\hline 2009 & & $4.48 \pm 0.02$ & $0.15 \pm 0.13$ & $0.37 \pm 0.29$ & $5 \pm 0.06$ \\
\hline 2010 & & $3.42 \pm 0.22$ & $0.18 \pm 0.15$ & $0.38 \pm 0.42$ & $3.99 \pm 0.79$ \\
\hline 2011 & & $2.94 \pm 0.12$ & $0.09 \pm 0.00$ & $0.09 \pm 0.01$ & $3.12 \pm 0.12$ \\
\hline
\end{tabular}




\begin{tabular}{|l|l|l|l|l|}
\hline 2012 & $3.50 \pm 0.31$ & $0.1 \pm 0.00$ & $0.12 \pm 0.02$ & $3.72 \pm 0.34$ \\
\hline 2013 & $4.19 \pm 0.10$ & $0.90 \pm 0.01$ & $0.92 \pm 0.03$ & $6.01 \pm 0.07$ \\
\hline 2014 & $4.97 \pm 0.37$ & $0.89 \pm 0.03$ & $0.99 \pm 0.19$ & $6.85 \pm 0.58$ \\
\hline 2015 & $4.50 \pm 0.13$ & $0.90 \pm 0.01$ & $0.87 \pm 0.02$ & $6.27 \pm 0.16$ \\
\hline 2016 & $4.37 \pm 0.73$ & $0.88 \pm 0.01$ & $0.85 \pm 0.03$ & $6.11 \pm 0.76$ \\
\hline
\end{tabular}

101

102 Table S6. Lake Superior measured concentration of each diastereomer and $\sum$ HBCDDs in ng/g wet 103 weight.

\begin{tabular}{|c|c|c|c|c|c|}
\hline $\begin{array}{l}\text { Lake } \\
\text { Superior }\end{array}$ & Year & $\begin{array}{l}\alpha-H B C D D \\
(n g / g ~ w w)\end{array}$ & $\begin{array}{l}\beta-H B C D D \\
(n g / g ~ w w)\end{array}$ & $\begin{array}{l}\gamma \text {-HBCDD } \\
(n g / g w w)\end{array}$ & $\begin{array}{l}\sum \text { HBCDDs } \\
\text { (ng/g ww) }\end{array}$ \\
\hline 1978 & & $2.36 \pm 0.05$ & $1.77 \pm 0.03$ & $1.57 \pm 0.03$ & $5.70 \pm 0.11$ \\
\hline 1986 & & $2.48 \pm 0.12$ & $1.81 \pm 0.01$ & $1.41 \pm 0.03$ & $5.69 \pm 0.14$ \\
\hline 1992 & & $3.79 \pm 0.14$ & $1.76 \pm 0.01$ & $1.63 \pm 0.12$ & $7.19 \pm 0.17$ \\
\hline 2004 & & $5.86 \pm 0.34$ & $1.80 \pm 0.04$ & $1.73 \pm 0.09$ & $9.38 \pm 0.34$ \\
\hline 2005 & & $4.93 \pm 0.16$ & $1.62 \pm 0.56$ & $2.18 \pm 0.95$ & $8.73 \pm 1.55$ \\
\hline 2006 & & $6.82 \pm 0.17$ & $1.93 \pm 0.07$ & $2.45 \pm 0.42$ & $11.2 \pm 0.66$ \\
\hline 2007 & & $4.37 \pm 0.23$ & $1.91 \pm 0.02$ & $2.06 \pm 0.16$ & $8.34 \pm 0.16$ \\
\hline 2008 & & $8.56 \pm 0.22$ & $1.89 \pm 0.05$ & $2.64 \pm 0.25$ & $13.1 \pm 0.52$ \\
\hline 2009 & & $3.93 \pm 0.19$ & $1.77 \pm 0.02$ & $1.37 \pm 0.02$ & $7.07 \pm 0.19$ \\
\hline 2010 & & $8.63 \pm 0.26$ & $1.91 \pm 0.06$ & $2.40 \pm 0.23$ & $12.9 \pm 0.48$ \\
\hline 2011 & & $3.72 \pm 0.48$ & $1.88 \pm 0.07$ & $1.91 \pm 0.11$ & $7.51 \pm 0.53$ \\
\hline 2012 & & $5.04 \pm 0.23$ & $1.87 \pm 0.05$ & $2.05 \pm 0.14$ & $8.96 \pm 0.33$ \\
\hline 2013 & & $3.37 \pm 0.27$ & $1.84 \pm 0.06$ & $1.91 \pm 0.10$ & $7.12 \pm 0.18$ \\
\hline 2014 & & $6.79 \pm 0.77$ & $1.88 \pm 0.01$ & $2.05 \pm 0.04$ & $10.7 \pm 0.74$ \\
\hline
\end{tabular}




\begin{tabular}{|l|l|l|l|l|}
\hline 2015 & $4.49 \pm 0.88$ & $1.60 \pm 0.45$ & $2.13 \pm 0.31$ & $8.22 \pm 0.84$ \\
\hline 2016 & $2.93 \pm 0.11$ & $1.83 \pm 0.02$ & $1.75 \pm 0.03$ & $6.51 \pm 0.16$ \\
\hline
\end{tabular}

104

105 Table S7. Lake Huron measured concentration of each diastereomer and $\sum$ HBCDDs in ng/g wet weight.

\begin{tabular}{|c|c|c|c|c|c|}
\hline Lake Huron & Year & $\begin{array}{l}\alpha-H B C D D \\
(n g / g w w)\end{array}$ & $\begin{array}{l}\beta-H B C D D \\
(n g / g ~ w w)\end{array}$ & $\begin{array}{l}\gamma \text {-HBCDD } \\
(n g / g \text { ww) }\end{array}$ & $\begin{array}{l}\sum \text { HBCDDs } \\
(\mathrm{ng} / \mathrm{g} \mathrm{ww})\end{array}$ \\
\hline 1978 & & $3.97 \pm 0.26$ & $1.37 \pm 0.03$ & $1.95 \pm 0.04$ & $7.29 \pm 0.30$ \\
\hline 1986 & & $3.31 \pm 0.29$ & $1.39 \pm 0.03$ & $1.65 \pm 0.08$ & $6.35 \pm 0.27$ \\
\hline 1992 & & $3.19 \pm 0.05$ & $1.39 \pm 0.02$ & $1.59 \pm 0.02$ & $6.17 \pm 0.09$ \\
\hline 2004 & & $5.92 \pm 0.79$ & $0.26 \pm 0.11$ & $0.91 \pm 0.09$ & $7.09 \pm 0.92$ \\
\hline 2005 & & $4.46 \pm 0.01$ & $0.30 \pm 0.14$ & $0.81 \pm 0.00$ & $5.57 \pm 0.13$ \\
\hline 2006 & & $6.23 \pm 0.73$ & $0.20 \pm 0.00$ & $1.07 \pm 0.28$ & $7.50 \pm 1.01$ \\
\hline 2007 & & $5.27 \pm 0.09$ & $0.40 \pm 0.00$ & $0.99 \pm 0.01$ & $6.66 \pm 0.08$ \\
\hline 2008 & & $5.69 \pm 0.44$ & $0.40 \pm 0.01$ & $0.65 \pm 0.11$ & $6.74 \pm 0.37$ \\
\hline 2009 & & $7.13 \pm 1.30$ & $0.60 \pm 0.01$ & $1.62 \pm 0.11$ & $9.35 \pm 1.40$ \\
\hline 2010 & & $5.39 \pm 0.01$ & $0.40 \pm 0.00$ & $0.99 \pm 0.00$ & $6.78 \pm 0.01$ \\
\hline 2011 & & $10.56 \pm 0.59$ & $0.46 \pm 0.12$ & $1.64 \pm 0.66$ & $12.7 \pm 1.04$ \\
\hline 2012 & & $7.47 \pm 0.44$ & $0.39 \pm 0.01$ & $1.47 \pm 0.17$ & $9.33 \pm 0.62$ \\
\hline 2013 & & $9.31 \pm 0.20$ & $0.39 \pm 0.01$ & $2.12 \pm 0.09$ & $11.8 \pm 0.21$ \\
\hline 2014 & & $6.33 \pm 0.33$ & $0.39 \pm 0.01$ & $1.73 \pm 0.77$ & $8.45 \pm 0.43$ \\
\hline 2015 & & $9.98 \pm 0.88$ & $0.39 \pm 0.00$ & $3.30 \pm 0.31$ & $13.7 \pm 0.95$ \\
\hline 2016 & & $9.08 \pm 0.23$ & $0.39 \pm 0.01$ & $1.69 \pm 0.11$ & $11.2 \pm 0.33$ \\
\hline
\end{tabular}


107 Table S8. Lake Erie measured concentration of each diastereomer and $\sum$ HBCDDs in ng/g wet weight.

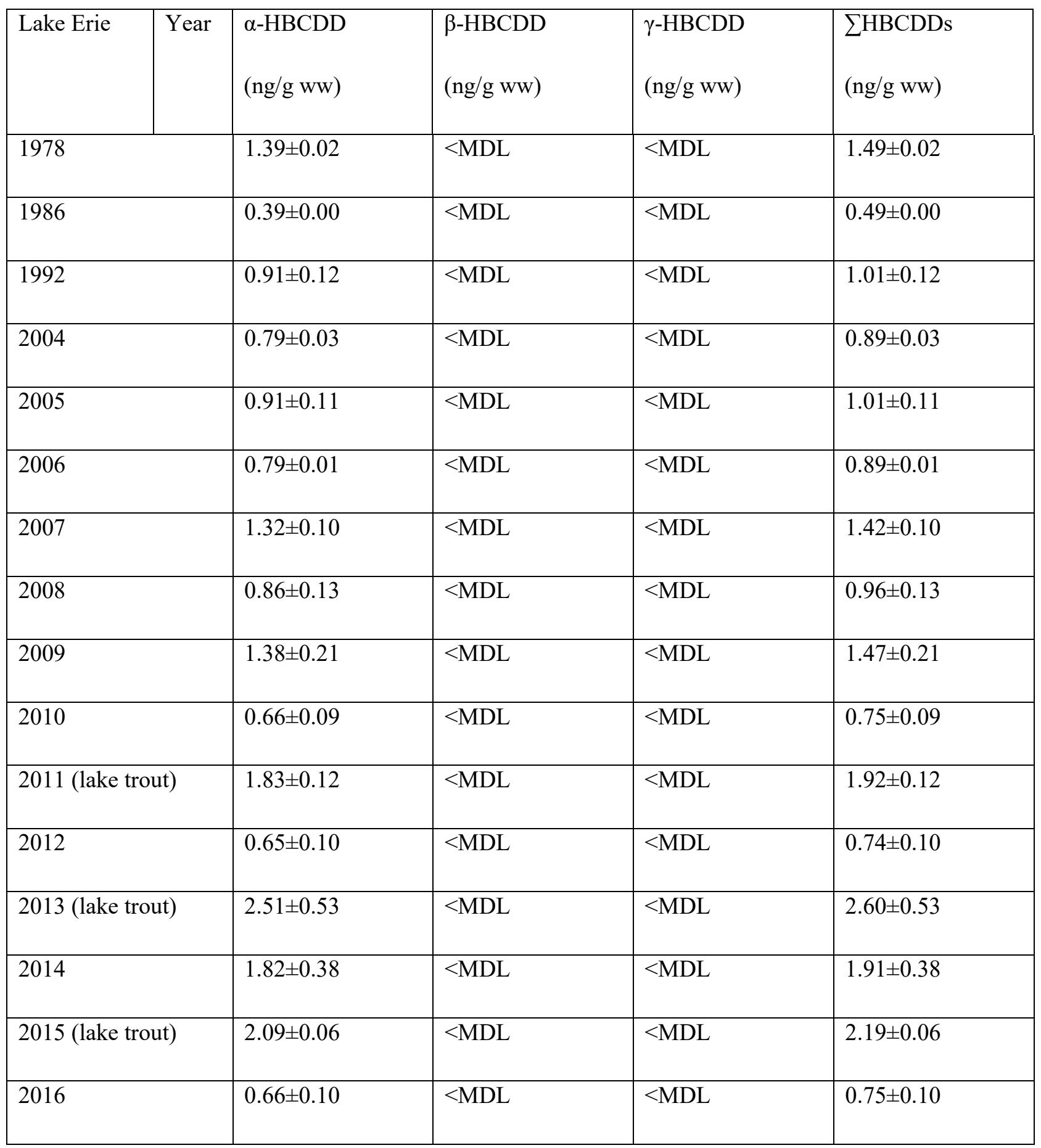


110 Results are presented in Table S8. Results below 0.05 and below 0.01 indicates significant and very 111 significant statistical differences respectively.

112 Table S9. HBCDD diastereomer distribution similarities between the Great Lakes (1978-2016).

\begin{tabular}{|l|l|}
\hline diastereomer & Test, $p$ value \\
\hline$\alpha$-HBCDD & One-way ANOVA, $<0.01$ \\
\hline$\beta$-HBCDD & Kruskal-Wallis One-way ANOVA on ranks, $<0.001$ \\
\hline$\gamma$-HBCDD & Kruskal-Wallis One-way ANOVA on ranks, $<0.001$ \\
\hline
\end{tabular}

113

114 Table S10. Tukey-Kramer analysis for $\alpha$-HBCDD distribution similarities between the Great Lakes 115 (1978-2016).

\begin{tabular}{|l|l|}
\hline Lakes & Tukey-Kramer, $p$ value \\
\hline LM vs LO & 0.93 \\
\hline LM vs LH & 0.48 \\
\hline LM vs LS & $<0.05$ \\
\hline LM vs LE & $<0.001$ \\
\hline LO vs LH & 0.92 \\
\hline LO vs LS & $<0.05$ \\
\hline LO vs LE & $<0.001$ \\
\hline LH vs LS & $<0.001$ \\
\hline
\end{tabular}

116

117 Table S11. Tukey-Kramer analysis for $\beta$-HBCDD distribution similarities between the Great Lakes 118 (1978-2016).

\begin{tabular}{|l|l|}
\hline Lakes & Tukey-Kramer, $p$ value \\
\hline
\end{tabular}




\begin{tabular}{|l|l|}
\hline LM vs LO & 0.13 \\
\hline LM vs LH & $<0.05$ \\
\hline LM vs LS & 0.81 \\
\hline LO vs LH & 0.78 \\
\hline LO vs LS & $<0.05$ \\
\hline LH vs LS & $<0.001$ \\
\hline
\end{tabular}

120 Table S12. Tukey-Kramer analysis for $\gamma$-HBCDD distribution similarities between the Great Lakes 121 (1978-2016).

\begin{tabular}{|l|l|}
\hline Lakes & Tukey-Kramer, $p$ value \\
\hline LM vs LO & 0.97 \\
\hline LM vs LH & 0.22 \\
\hline LM vs LS & 0.14 \\
\hline LO vs LH & 0.05 \\
\hline LO vs LS & $<0.05$ \\
\hline LH vs LS & $1 *$ \\
\hline
\end{tabular}

* $\mathrm{p}$ value equal to one in this test is treated as "no significant difference is found between the two ranks".

(1) Pagano, J. J.; Garner, A. J.; McGoldrick, D.; Crimmins, B. S.; Hopke, P. K.; Milligan, M. S.; Holsen, T. M. Age-Corrected Trends and Toxic Equivalence of PCDD/F and CP-PCBs in Lake Trout and Walleye From the Great Lakes: 2004-2014. Environ. Sci. Technol. 2017, 52 (2), 712-721.

(2) Zhou, C.; Cohen, M. D.; Crimmins, B. A.; Zhou, H.; Johnson, T. A.; Hopke, P. K.; Holsen, T. M. Mercury Temporal Trends in Top Predator Fish of the Laurentian Great Lakes from 2004 to 2015: Are Concentrations Still Decreasing? Environ. Sci. Technol. 2017, 51 (13), 7386-7394. https://doi.org/10.1021/acs.est.7b00982. 